\title{
硅盐互作下小獐毛植物体内元素分布 及生理特性的研究
}

\author{
陈 阳 ${ }^{1,2,3}$ 王 贺 3 张福锁 $13 *$ 贾恢先 $^{2}$ \\ (1 中国科学院新疆生态与地理研究所,乌鲁木齐 830011) \\ （2 甘肃农业大学农学系 兰州 730070）（3 中国农业大学资源环境学院, 北京 100094)
}

摘 要 以新疆泌盐植物小獐毛 (Aeluropus pungens) 为材料, 研究盐胁迫下小獐毛植物体元素吸收、分泌和过氧化物 酶活性的变化以及硅对上述指标的影响。结果表明:在盐分胁迫下, 其植物体钠离子浓度升高, 钙元素含量降低, 其它元素含量变化不明显。叶片硅元素含量随盐胁迫而增加。同时, 当盐分浓度由 $0 \mathrm{mmol} \mathrm{L}^{-1}$ 升至 $120 \mathrm{mmol} \mathrm{L}^{-1}$ 浓度时, 盐腺对各种离子的分泌作用表现为先加强 $\left(60 \mathrm{mmol} \mathrm{L} \mathrm{L}^{-1}\right)$ 后降低 $\left(120 \mathrm{mmol} \mathrm{L} \mathrm{L}^{-1}\right)$ 的趋势; 其叶及根可溶性 蛋白变化不明显, 但过氧化物酶活性随盐胁迫而升高; 可溶性蛋白含量叶片高于根部, 而过氧化物酶活性根部高于 叶片。同时盐胁迫对小獐毛根部及叶片含水量无明显影响。通过细胞化学定位结果显示, 小獐毛叶表富含硅元 素, 硅元素在叶表排列沿叶脉方向呈线性分布; 在其叶片盐腺上, 硅元素分布于帽细胞顶部, 在此过氧化物酶 (POD) 活性也较强。元素分析结果显示, 小獐毛体内富积硅元素。在较低的 $\mathrm{NaCl}\left(60 \mathrm{mmol} \mathrm{L} \mathrm{L}^{-1}\right)$ 浓度下, 施用硅处理可减 少叶片钠离子浓度, 使地上部对钾、钠元素的相对选择性明显提高。在较高盐浓度 $\left(120 \mathrm{mmol} \mathrm{L} \mathrm{L}^{-1} \mathrm{NaCl}\right)$ 下, 加硅对 叶片钠离子浓度的降低作用效果不明显。在盐胁迫下, 加硅未能减少根中钠离子浓度, 但可明显增加叶片 POD 活 性。实验结果表明, 盐生植物小獐毛可通过维持体内含水量, 调节植物体内元素分布以及增加 POD 活性适应一定 程度的盐胁迫。同时低盐条件下施硅处理小獐毛根系培养环境可通过减少盐分向地上部的运输增加叶片清除自 由基的能力从而提高植物抗盐性。

关键词 盐胁迫 硅 细胞化学定位 盐腺 过氧化物酶

\section{THE EFFECTS OF SILICON ON IONIC DISTRIBUTION AND PHYSIOLOGICAL CHARACTERISTIC OF AELUROPUS PUNGENS UNDER SALINITY CONDITIONS}

\author{
CHEN Yang ${ }^{1,3}$ WANG He ${ }^{3}$ ZHANG Fu-Suo ${ }^{13}$ and JIA Hui-Xian ${ }^{2}$ \\ (1 Xinjiang Institute of Ecology and Geography, the Chinese Academy of Sciences , Urumqi 830011 , China) \\ (2 Department of Agronomy , Gansu Agricultral University, Lanzhou 730070 , China) \\ (3 College of National Resources and Environmental Sciences , China Agricultrue University , Beijing 100094 , China)
}

\begin{abstract}
The changes of ionic absorption, secretion and the activity of POD on Aeluropus pungens under salinity conditions and the effects of silicon nutrition on those indices were studied using light microscopy , cytochemical localization, and quantitative and qualitative analysis for ions and physiological indices. The results were as follows : Under salinity conditions , the $\mathrm{Na}^{+}$content increased and the $\mathrm{Ca}^{2+}$ content of the plant tissues decreased, while the other ions remained constant, and the concentrations of silicon in leaves increased with increasing salinity. The rate of ion secretion increased between 0 and $60 \mathrm{mmol} \mathrm{L} \mathrm{L}^{-1} \mathrm{NaCl}$, and then declined when the $\mathrm{NaCl}$ concentrations in the roots reached $120 \mathrm{mmol} \mathrm{L}^{-1}$. The content of soluble protein was unaffected but the POD activity was enhanced by the salt treatment. The amount of soluble proteins were higher in the roots than that in the leaves, while on the POD activity, the situation was the contrast. The water content did

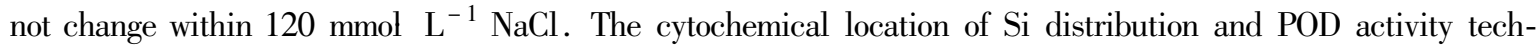
niques showed silica was distributed in rows along the leaf veins on the leaf surfaces, and some traces on the papillaes. On the salt glands $\mathrm{Si}$ accumulated on the top of the cap cell where the strong POD activity was found. By analyzing the elements, it was found that silicon tended to accumulate on the tissues of Aeluropus pungens. The $\mathrm{Na}$ content and the selective of $\mathrm{Na}, \mathrm{K}$ of the shoots, which was not affected under $120 \mathrm{mmol}$ $\mathrm{L}^{-1} \mathrm{NaCl}$, was reduced under $60 \mathrm{mmol} \mathrm{L}{ }^{-1} \mathrm{NaCl}$ by the addition of $3 \mathrm{mmol} \mathrm{L} \mathrm{L}^{-1} \mathrm{Na}_{2} \mathrm{SiO}_{3}$. However , the $\mathrm{Na}$
\end{abstract}


content remained unaffected in the roots by the addition of Silicon to both salt concentrations. The above experiments demonstrated that halophyte Aeluropus pungens adapted to salinity conditions by adjusting water conditions , the ion distribution and POD activity on the plant tissues. The capacity of salt tolerance was improved , which was due to the inhibition of the Na transport from roots to shoots and the increasing abilities of antioxidative defense when silicon was added to the nutrient solutions.

Key words Salt stress, Silicon, Cyto-chemcial location , Salt gland , Peroxidase

土壤盐渍化目前是限制农业、林业的主要因素 之一。据报道，在世界范围内，近 23 亿公顷灌溉土 地的 $1 / 3$ 受到盐分胁迫。并且过量的灌溉及降雨的 缺乏加剧了盐化程度, 使土壤盐渍化面积逐年增加。 盐分胁迫不仅发生在水分蒸发超过降雨量的干旱及 半干旱地区, 在湿润地区尤其是沿海地区也时有发 生(Ehman et al .,1996)。因此了解盐生植物结构和 功能对于盐生境的适应性, 将有助于充分利用盐生 植物资源, 开发盐碱地的生产潜力。

生长于盐碱地的泌盐植物可通过调节盐环境下 植物体的矿质平衡、保持植物组织中低浓度的盐分 适应盐生环境(Marcum \& Murdoch ,1990)。其盐腺对 于盐分的分泌作用是其抵制盐害的重要机制之一。 从盐分分泌结构上, Melissa 等 (1992)通过 X 能谱技 术证实了泌盐植物盐结晶不聚集在气孔下腔, 并指 出分泌盐分的部位是盐腺。单子叶植物泌盐结构盐 腺一般为双细胞构成, 双子叶植物盐腺由多细胞构 成。泌盐植物都拥有相似的超微结构和生理特征。 目前关于盐腺中钠、氯元素的运输途径及钠、氯元素 在细胞中的定位已有研究报道 (Storey \& Thomson , 1994）,但关于盐腺中其它元素的分布特点及酶的细 胞化学定位尚末见报道。从生理机能上,盐分分泌 与生长环境的盐浓度有一定的关系, 盐腺的分泌作 用是主动生理过程。同时盐腺对离子分泌具有选择 性 表现为分泌不同离子的选择性及植物种类对离 子分泌的选择性(Pollak \& Waisel ,1970)。在酶反应 方面, 大多数植物在盐胁迫下表现为抗氧化酶活性 的减低及自由基含量的增加 (Hernandez et al., 1993)。Flowers 等 (1977) 认为盐生植物与甜土植物 的酶系统对盐分具有同样的敏感性，因此对于盐生 植物酶在盐胁迫下反应的研究亦鲜有报道。

在土壤中硅含量仅次于氧, 硅在土壤溶液中主 要来源于硅酸, 硅以单硅酸的形式被植物根系吸收 及转移, 在植物体中通常以蛋白石的形式存在。硅 在植物中的含量与钙、镁、磷等大量元素相似, 有些 植物可大量积累硅, 通常在草中硅含量高于其它无 机成分。硅不仅有利于植物的生长发育, 在某种程 度上还可以保护植物抵制生物及非生物胁迫, 如硅
可加强内皮层细胞壁而减轻水分胁迫; 硅可以强化 细胞壁; 可能参与植物的代谢调节能力;由硅引发的 保护抵抗病原菌、昆虫等生物胁迫效果显著(Epstin， 1999)。在提高植物抗盐性方面，硅可缓解盐胁迫下 营养失衡及增加植物体对盐胁迫的抗性。在小麦及 大麦中, 盐胁迫下加硅处理使生物量提高, 钠的转运 受到影响 (Ahmad et al. ,1992 ; Liang, 1999)。Bradbury 和 Ahmad (1990) 认为在海水中添加 0.47 $\mathrm{mmol} \mathrm{L} \mathrm{L}^{-1} \mathrm{SiO}_{2}$ 可减少它对牧豆树 (Prosopis juliflora) 生长的抑制作用。但目前关于盐生植物硅与植物抗 盐性关系的研究鲜有报道。本研究选用生长于新疆 的盐生草甸、可作为优良牧草及草坪草的多年生丛 生禾本科植物小獐毛 (Aeluropus pungens) 为实验材 料，针对其属于泌盐植物这一适应盐渍生境的特点， 于 8 月中旬采集成熟的小獐毛种子, 采用室内水培 方法, 在研究盐腺过氧化物酶的酶学定位及定量结 果及其硅分布特点的基础上, 探索小獐毛适应盐生 境的元素分布、酶活性和水分关系特点，以及硅盐互 作对植物体元素分布、过氧化物酶活性的影响。

\section{1 材料和方法}

\section{1 材料的培养}

小獐毛种子于 2000 年 8 月采集于新疆阜康沙 漠生态站，于 2001 年 3 月播种于覆有纱布的聚乙烯 珠上, 出苗后以培养液培养, 待苗长至 $3 \mathrm{~cm}$ 高时移 栽至含培养液的塑料盆中, 上有含孔塑料盖, 每孔 10 株, 共 5 孔。培养液硅含量为 $0.057 \mathrm{mmol} \mathrm{L} \mathrm{L}^{-1}$ 。 $14 \mathrm{~d}$ 后分别进行加硅及缺硅处理。使硅酸钠浓度为

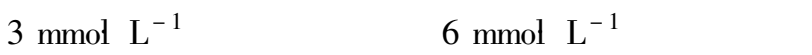
不同处理保持相同钠浓度。然后分别在上述培养液 中逐次加入 $0 \mathrm{mmol} \cdot \mathrm{L}^{-1}, 60 \mathrm{mmol} \cdot \mathrm{L}^{-1}, 120$ $\mathrm{mmol} \mathrm{L} \mathrm{L}^{-1} \mathrm{NaCl}$ 处理 $20 \mathrm{~d}$ 后测定以下指标。以上培 养液每 $3 \mathrm{~d}$ 更换 1 次。

\section{2 相关指标的测定}

\subsection{1 生物量及植物体的含水量的测定}

采用称重法测定生物量 ;参见赵可夫等 (1999) 提供的方法测定植物体的含水量。

1.2.2 泌盐速率测定 
参见 Marilyn 和 Ball (1988) 的方法, 以每天分泌 营养元素微摩尔数表示。

\subsection{3 矿质元素含量的测定}

分别取测定前后的植物根、茎、叶于 $90{ }^{\circ} \mathrm{C}$ 烘干 $48 \mathrm{~h}$ ，参见 Marcum(1994) 提供的方法制备钠、钾、钙、 镁、磷待测液。硅元素待测液以 Fridlund 等 (1994) 方法制备。以氢氟酸提取, 测定前以硼酸排除游离 的氢氟酸。元素含量采用 Perkin-Elmer Optima $3300 D V$ 等离子光谱进行分析。

\subsection{4 可溶蛋白及过氧化物酶的测定}

参见徐朗莱 (1989) 方法提取蛋白及过氧化物 酶。以牛血清蛋白为标准, 以考马斯亮蓝 G-250 染 色法测定蛋白质含量, 以单位植物干重所含蛋白质 的毫克数表示。参见 Mensen (1998) 方法测定过氧 化物酶活性，以每毫克蛋白所具有的比活性表示。

以上处理均设 3 次以上重复, 以 SAS 软件进行 统计分析。

\section{3 硅元素分布及过氧化物酶活性定位}

参见 Dayamandan 等 (1983) 提供的方法定位硅 元素分布并略加改进，以乙醇及丙酮将叶片脱色，加 拿大树胶封片观察。参见 Peter 等 (1972) 提供的方 法定位 POD 活性。

\section{2 结果与分析}

2.1 小獐毛生长状况及其植物体内水分含量的比 较

未受到盐分胁迫时，添加硅于培养环境中对小 獐毛生物量无明显影响, 但长势有所差别。表现为 加硅培养的植物地上部茎叶挺立, 而缺硅培养的植 物枝叶柔软，易倒。盐分胁迫抑制了植物生长，但加 硅培养增加了盐分胁迫下小獐毛的生物量 (图 1)。 并且随着盐分胁迫强度的提高，小獐毛叶表有盐分 分泌。

小獐毛在 0、60、120 mmol L-1氯化钠下, 不表现 出含水量的明显变化，外观良好，未见叶片枯焦及萎 蒸现象，说明其自身抗渗透胁迫低水势的机制特别 完善。在非盐及盐胁迫下施硅对小獐毛水分状况亦 无明显影响(图 2)。

以上结果说明小獐毛对盐胁迫有一定程度的耐 受力。由于盐分胁迫对植物体水分状况无影响，因 此添加硅对水分状况的调节作用不明显。但盐胁迫 下添加硅有明显增加植物体生物量的作用, 这可能 与硅降低了植物体内钠离子浓度有关。

2.2 小獐毛在不同盐浓度下元素含量的变化及加 硅对营养元素运输、分配的影响
由表 1 可以看出，小獐毛植物叶片元素含量以 硅元素、钾元素含量最高, 铁元素含量最低。随着培 养环境氯化钠浓度的升高, 植物体内钙元素含量降 低。铁、钾、磷和镁元素变化不明显。在 $60 \mathrm{mmol}$ $\mathrm{L}^{-1} \mathrm{NaCl}, 120 \mathrm{mmol} \mathrm{L}^{-1} \mathrm{NaCl}$ 胁迫下, 缺硅培养的植 物叶片和根部钠离子浓度分别升高了 $448 \% 、 552 \%$ ， $147 \% 、 375 \%$ 。而加硅培养的植物叶片和根部钠离 子浓度分别升高了 $248 \% 、 630 \%, 174 \% 、 391 \%$ 。小 獐毛地上部与地下部离子分布特点表现为: 钾、镁元 素地上部含量高于地下部, 铁、磷元素地下含量明显 高于地上部。在未加盐分处理时,根部钙元素含量 高于叶片; 在盐胁迫下, 根部钙元素含量低于叶片。 说明盐胁迫下钙元素相对地上部分配比例加大, 钙 元素有向地上运输的趋势。

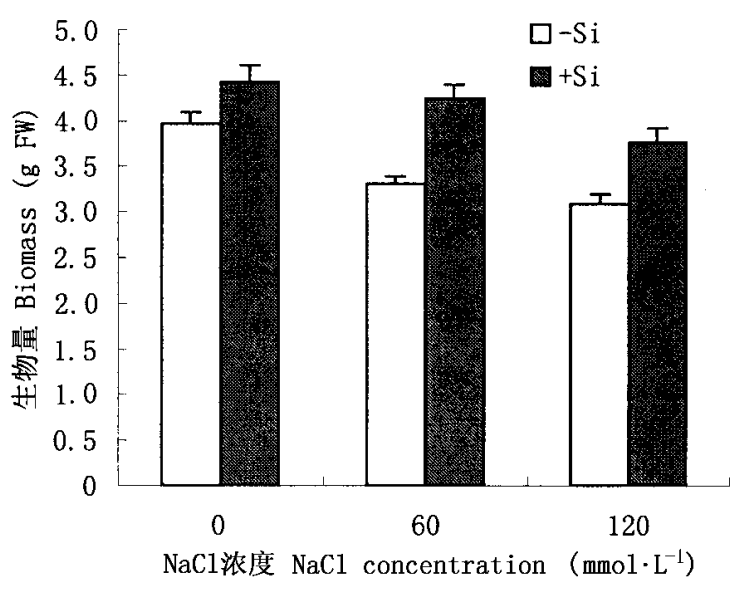

图 1 硅盐互作对小獐毛生物量的影响 (数据为 5 次测定结果的平均值 \pm 误差 $n=5$ ) Fig. 1 Effect of silicon on the biomass of Aeluropus pungens under salt stress (Data are means of 5 replication \pm SE $n=5$ )

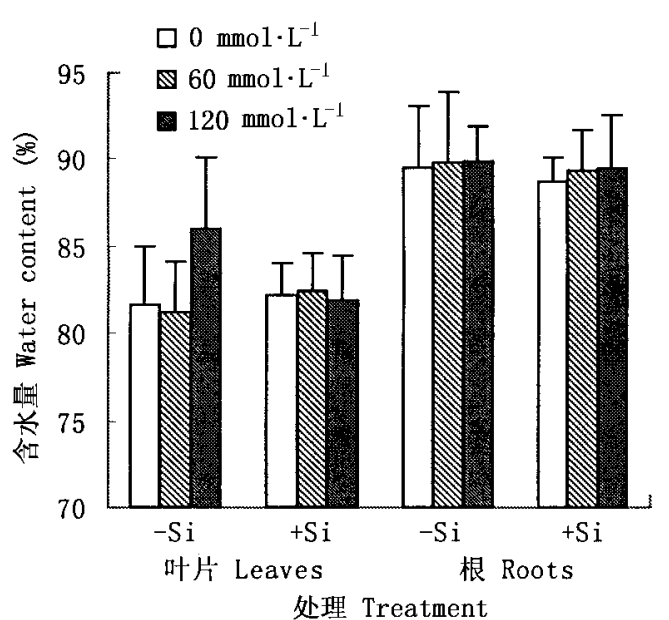

图 2 不同处理条件下叶片及根含水量的变化 (数据为 5 次测定结果的平均值 \pm 误差 $n=5$ )

Fig. 2 The changes of water content on leaves and roots under various treatments (Data are means of 5 replication \pm SE $n=5$ ) 
表 1 在 $0 、 60 、 120 \mathrm{mmol} \mathrm{L} \mathrm{L}^{-1} \mathrm{NaCl}$ 下硅对小獐毛不同部位离子含量的影响 $\left(\mathrm{mmol} \mathrm{g}^{-1} \mathrm{DW}\right)$

Table 1 The effects of silicon on the ions concentration of shoots and roots grown in $0 \mathrm{mmol} \mathrm{L}{ }^{-1} 、 60 \mathrm{mmol} \mathrm{L} \mathrm{L}^{-1}$ and $120 \mathrm{mmol} \mathrm{L} \mathrm{L}^{-1} \mathrm{NaCl}$

\begin{tabular}{|c|c|c|c|c|c|c|c|}
\hline \multirow{3}{*}{$\begin{array}{l}\text { 元素 } \\
\text { Ions }\end{array}$} & \multirow{3}{*}{$\begin{array}{c}\text { 部位 } \\
\text { Various parts }\end{array}$} & \multicolumn{6}{|c|}{$\mathrm{NaCl}$ 浓度 $\mathrm{NaCl}$ concentration $\left(\mathrm{mmol} \mathrm{L}^{-1}\right)$} \\
\hline & & \multicolumn{3}{|c|}{$-\mathrm{Si}$} & \multicolumn{3}{|c|}{$+\mathrm{Si}$} \\
\hline & & 0 & 60 & 120 & 0 & 60 & 120 \\
\hline \multirow[t]{2}{*}{$\mathrm{Fe}$} & 地上部 Shoot & $0.00318^{\mathrm{b}}$ & $0.00257^{\mathrm{b}}$ & $0.00209^{\mathrm{b}}$ & $0.00239^{\mathrm{b}}$ & $0.00214^{\mathrm{b}}$ & $0.00182^{b}$ \\
\hline & 根 Root & $0.229^{\mathrm{a}}$ & $0.285^{\mathrm{a}}$ & $0.278^{\mathrm{a}}$ & $0.136^{\mathrm{a}}$ & $0.219^{\mathrm{a}}$ & $0.251^{\mathrm{a}}$ \\
\hline \multirow[t]{2}{*}{$\mathrm{K}$} & 地上部 Shoot & $0.821^{\mathrm{a}}$ & $0.775^{\mathrm{a}}$ & $0.777^{\mathrm{a}}$ & $0.633^{\mathrm{a}}$ & $0.695^{\mathrm{a}}$ & $0.705^{\mathrm{a}}$ \\
\hline & 根 Root & $0.586^{\mathrm{ab}}$ & $0.685^{\mathrm{a}}$ & $0.523^{\mathrm{b}}$ & $0.498^{\mathrm{b}}$ & $0.509^{\mathrm{b}}$ & $0.518^{\mathrm{b}}$ \\
\hline \multirow[t]{2}{*}{$\mathrm{P}$} & 地上部 Shoot & $0.125^{\mathrm{b}}$ & $0.139^{\mathrm{b}}$ & $0.125^{\mathrm{b}}$ & $0.105^{\mathrm{b}}$ & $0.112^{\mathrm{b}}$ & $0.115^{\mathrm{b}}$ \\
\hline & 根 Root & $0.459^{\mathrm{a}}$ & $0.538^{\mathrm{a}}$ & $0.442^{\mathrm{a}}$ & $0.327^{\mathrm{a}}$ & $0.425^{\mathrm{a}}$ & $0.473^{\mathrm{a}}$ \\
\hline \multirow[t]{2}{*}{$\mathrm{Na}$} & 地上部 Shoot & $0.062^{c}$ & $0.340^{\mathrm{ab}}$ & $0.404^{\mathrm{a}}$ & $0.062^{\mathrm{c}}$ & $0.216^{\mathrm{b}}$ & $0.453^{\mathrm{a}}$ \\
\hline & 根 Root & $0.097^{\mathrm{c}}$ & $0.240^{\mathrm{b}}$ & $0.461^{\mathrm{a}}$ & $0.087^{\mathrm{c}}$ & $0.238^{\mathrm{b}}$ & $0.427^{\mathrm{a}}$ \\
\hline \multirow[t]{2}{*}{$\mathrm{Ca}$} & 地上部 Shoot & $0.198^{\mathrm{ab}}$ & $0.175^{\mathrm{ab}}$ & $0.124^{\mathrm{b}}$ & $0.174^{\mathrm{ab}}$ & $0.163^{\mathrm{ab}}$ & $0.145^{\mathrm{b}}$ \\
\hline & 根 Root & $0.212^{\mathrm{ab}}$ & $0.097^{\mathrm{c}}$ & $0.075^{\mathrm{c}}$ & $0.176^{\mathrm{ab}}$ & $0.105^{\mathrm{c}}$ & $0.097^{\mathrm{c}}$ \\
\hline \multirow[t]{2}{*}{$\mathrm{Mg}$} & 地上部 Shoot & $0.132^{\mathrm{a}}$ & $0.118^{\mathrm{a}}$ & $0.103^{\mathrm{a}}$ & $0.110^{\mathrm{a}}$ & $0.100^{\mathrm{a}}$ & $0.104^{\mathrm{a}}$ \\
\hline & 根 Root & $0.115^{\mathrm{a}}$ & $0.083^{\mathrm{b}}$ & $0.074^{\mathrm{b}}$ & $0.052^{\mathrm{b}}$ & $0.079^{\mathrm{b}}$ & $0.055^{\mathrm{b}}$ \\
\hline \multirow[t]{2}{*}{$\mathrm{Si}$} & 地上部 Shoot & $0.330^{\mathrm{d}}$ & $0.401^{\mathrm{d}}$ & $0.389^{\mathrm{d}}$ & $0.901^{\mathrm{b}}$ & $1.257^{\mathrm{ab}}$ & $1.091^{\mathrm{b}}$ \\
\hline & 根 Root & $0.462^{\mathrm{d}}$ & $0.762^{\mathrm{c}}$ & $0.633^{\mathrm{c}}$ & $1.991^{\mathrm{a}}$ & $1.363^{\mathrm{ab}}$ & $0.999^{b}$ \\
\hline
\end{tabular}

同一列相同字母表示差异未达显著水平 The same letter within a column means no significant at $95 \%$ probability $(p>0.05)$

施用硅元素与根系培养环境中，对铁、钾、磷、 钙、镁元素含量无影响，但增加硅元素在叶片及根的 含量。而在 $60 \mathrm{mmol} \mathrm{L}^{-1} \mathrm{NaCl}$ 下, 叶片钠元素含量 可减少 $36.47 \%$ 。在较高盐浓度 $\left(120 \mathrm{mmol}^{-} \mathrm{L}^{-1}\right.$ $\mathrm{NaCl})$ 下,加硅对叶片钠元素作用效果不明显。而在 $60 \mathrm{mmol} \mathrm{L} \mathrm{L}^{-1} \mathrm{NaCl}$ 及 $120 \mathrm{mmol} \mathrm{L} \mathrm{L}^{-1} \mathrm{NaCl}$ 浓度下,加硅 未减少根中钠元素含量。该结果暗示硅的作用仅限 于一定浓度盐分处理, 且其作用方式为减少钠离子 向地上的运输。

\section{3 不同盐浓度下施硅对离子分泌的影响(表 2)}

小獐毛盐腺可分泌钠、钾、钙、镁、磷、铁离子。 而在盐分胁迫下，小獐毛对钠离子的分泌作用最强， 其次是钾离子，对其它离子的分泌表现为钙 > 镁 >

表 2 硅盐互作对离子分泌量的影响 $\left(\mu \mathrm{mol} \mathrm{g} \mathrm{g}^{-1} \mathrm{DW}\right)$

Table 2 The effects of additional silicon on the ions secretion under various $\mathrm{NaCl}$ concentration

\begin{tabular}{ccccc}
\hline 元素 & 处理 & \multicolumn{3}{c}{$\mathrm{NaCl}$ 浓度 $\mathrm{NaCl}$ concentrations $\left(\mathrm{mmol} \mathrm{L}^{-1}\right)$} \\
\cline { 3 - 5 } Ions & Treatment & 0 & 60 & 120 \\
\hline $\mathrm{Fe}$ & $-\mathrm{Si}$ & $0.056^{\mathrm{ab}}$ & $0.285^{\mathrm{b}}$ & $0.039^{\mathrm{b}}$ \\
& $+\mathrm{Si}$ & $0.116^{\mathrm{a}}$ & $0.056^{\mathrm{ab}}$ & $0.039^{\mathrm{b}}$ \\
$\mathrm{K}$ & $-\mathrm{Si}$ & $107.8^{\mathrm{bc}}$ & $201.8^{\mathrm{ab}}$ & $86.59^{\mathrm{c}}$ \\
& $+\mathrm{Si}$ & $241.4^{\mathrm{a}}$ & $175.5^{\mathrm{b}}$ & $95.26^{\mathrm{c}}$ \\
$\mathrm{P}$ & $-\mathrm{Si}$ & $1.019^{\mathrm{c}}$ & $5.903^{\mathrm{a}}$ & $1.600^{\mathrm{bc}}$ \\
& $+\mathrm{Si}$ & $1.484^{\mathrm{bc}}$ & $4.484^{\mathrm{ab}}$ & $1.526^{\mathrm{bc}}$ \\
$\mathrm{Na}$ & $-\mathrm{Si}$ & $34.57^{\mathrm{d}}$ & $1438^{\mathrm{a}}$ & $855.7^{\mathrm{b}}$ \\
& $+\mathrm{Si}$ & $65.93^{\mathrm{c}}$ & $505.7^{\mathrm{bc}}$ & $666.1^{\mathrm{b}}$ \\
$\mathrm{Ca}$ & $-\mathrm{Si}$ & $23.28^{\mathrm{b}}$ & $47.18^{\mathrm{a}}$ & $19.98^{\mathrm{b}}$ \\
& $+\mathrm{Si}$ & $40.98^{\mathrm{a}}$ & $33.10^{\mathrm{ab}}$ & $22.03^{\mathrm{b}}$ \\
$\mathrm{Mg}$ & $-\mathrm{Si}$ & $12.54^{\mathrm{b}}$ & $20.33^{\mathrm{ab}}$ & $9.917^{\mathrm{c}}$ \\
& $+\mathrm{Si}$ & $28.63^{\mathrm{a}}$ & $16.33^{\mathrm{b}}$ & $9.458^{\mathrm{c}}$ \\
\hline
\end{tabular}

通过一维方差分析, 具有不同字母的处理差异显著 The same letter within a column means no significant at $95 \%$ probability $(p>0.05)$
磷 $>$ 铁。并且盐胁迫增加了盐腺对钠、磷元素的分 泌量。在 $60 \mathrm{mmol} \mathrm{L}^{-1} \mathrm{NaCl}$ 处理下,小獐毛盐腺对 钠离子的分泌增加量远高于对其它各离子分泌的增 加程度。

未加盐分处理下,加硅增加盐腺对各种离子的 分泌量。在 $60 \mathrm{mmol} \mathrm{L}^{-1} \mathrm{NaCl}$ 下, 盐胁迫下加硅可 减少盐腺对钠、钾、钙、镁、磷、铁的分泌。但当盐浓 度超过 $120 \mathrm{mmol} \mathrm{L} \mathrm{L}^{-1} \mathrm{NaCl}$ 时，加硅对离子分泌量无 明显影响。

缺硅培养植物其培养环境盐分浓度增加 1 倍时 (60 $\left.120 \mathrm{mmol} \cdot \mathrm{L}^{-1} \mathrm{NaCl}\right)$, 钠离子分泌减少了 $40.5 \%$ 植物体钠离子浓度增加了 $18.7 \%$;加硅处 理 培养液盐浓度增加 1 倍时, 钠离子分泌末见明显 减少, 而植物体钠离子浓度却增加 2.1 倍。可见植 物体内钠离子浓度除受到钠离子分泌作用影响以 外，在很大程度上还受到其它因素的限制。

2.4 盐胁迫下加硅处理时小獐毛叶片及根 $\mathrm{Na} / \mathrm{K}$ 、 盐分分必 $\mathrm{Na} / \mathrm{K}$ 以及 $\mathrm{Na}$ 地上/ $\mathrm{Na}$ 地下的变化

由钠、钾比可以看出,随着氯化钠浓度的升高， 植物根、叶对钠的吸收选择性增强, 对钾的选择性相 对降低。并且植物体内低于盐分分泌汁液中的 $\mathrm{Na}$ / $\mathrm{K}$ 。说明盐腺对于离子的分泌具有选择性。盐胁迫 下加硅, 在较低的 $\mathrm{NaCl}\left(60 \mathrm{mmol} \mathrm{L}^{-1}\right)$ 浓度下, 地上 部对钠的选择性明显降低，增加对钾元素吸收的选 择性。根对钠的选择性无显著变化。盐胁迫下加硅 降低盐分泌液中 $\mathrm{Na} / \mathrm{K}$, 表现为对钠离子的分泌选择 性降低。由钠地上含量与地下含量比值可以看出, $60 \mathrm{mmol} \mathrm{L}{ }^{-1} \mathrm{NaCl}$ 浓度下加硅减少了钠元素向地上 
部的分配比例。而在 $120 \mathrm{mmol} \mathrm{L} \mathrm{L}^{-1} \mathrm{NaCl}$ 浓度下，加 硅对叶代谢有利于提高抗逆性待协调节有重要作 用。输、根钠钾比及钠元素在地上及地下分布比例 均无显著影响。因此盐胁迫下加硅降低地上部钠离 子浓度含量与其减少盐分运输有较大关系, 而与盐 分分泌关系不大。即低盐条件下硅缓解盐害的意义 在于减少盐分由根向地上部运输的作用(表 3 )。

2.5 盐胁迫下加硅处理时小獐毛叶片及根可溶蛋 白及过氧化物酶活性的变化

在盐胁迫下, 可溶蛋白含量无明显变化, 使用硅 对可溶蛋白含量无明显影响。盐胁迫下叶片及根 POD 活性升高。盐胁迫下施用硅, 可分别增加叶片 及根 POD 活性的 $53.49 \% 、 5.21 \%$ 。其中在叶片中 的蛋白质含量高于根，而根部 POD 活性高于叶片 (表 4)。以上结果暗示盐胁迫下加硅可有效地提高 叶片清除自由基的能力。

2.6 小獐毛叶片硅及过氧化物沉积

小獐毛叶表富含硅元素, 表皮毛含丰富的硅元 素。硅元素在叶表排列沿叶脉方向呈线性分布, 形 状哑铃形(图 3A ,B)。其叶片盐腺由 1 个基细胞及 1 个突起于表皮上的帽细胞组成, 硅元素分布于分泌 细胞的帽细胞顶部,在此 POD 活性也较强, 它们沉 积与分布的部位呈现很好的一致性(图 4A ,B)。这 暗示硅元素的沉积可能与 POD 活性有关, 同时硅元
素在帽细胞的沉积程度可能有参与调节元素分泌的 作用。

\section{3 讨 论}

盐分影响植物生长原因来自于离子毒害这一直 接因素及土壤盐离子对土壤水势所造成的间接影 响。盐分可造成由于渗透调节能力的缺乏而引起的 植物吸水受到抑制以及生理干旱 (Schilmper, 1986 ; Flowers et al. , 1986)。而过量的钠离子含量能导致 植物细胞质膨胀变化, 破坏了质膜选择透性, 使细胞 内离子大量流入细胞外 影响一些酶的结构与功能， 破坏细胞的新陈代谢 (Marcum，1999)。本研究结果 显示, 小獐毛抗盐有 3 个主要方面: 1 ) 抗渗透胁迫。 在盐逆境条件下, 为了避免在高盐条件下渗透失衡, 盐生植物可以从外界环境中吸收积累钾、钠、氯等无 机离子 经离子区域化以减轻盐离子的毒害作用, 也 可以合成、聚集非毒性细胞质中相容性物质，如脯氨 酸, 甜菜碱等进行渗透调节作用。通常以上两种途 径并存, 只是不同的植物, 器官和组织所占的比重不 同。Marcum (1994) 研究结果显示海滨雀稗草 (Paspalum seashore) 和奥古氏草 ( Stenotaphrum augustinegrasst $)$ 等草坪植物在盐胁迫下存在渗透调节及维持 正常的膨压的能力。Marcum 和 Mardoch (1990) 认为 在 $\mathrm{C}_{4}$ 草坪草中 盐胁迫下其生存与盐离子聚集相关

表 3 硅盐互作对小獐毛 $\mathrm{Na}$ 地上/ $\mathrm{Na}$ 地下叶片、根和盐分分泌液中 $\mathrm{Na} / \mathrm{K}$ 的影响

Table $3 \mathrm{Na}_{\text {shoot }} / \mathrm{Na}_{\text {root }}, \mathrm{Na} / \mathrm{K}$ on leaves and roots、 $\mathrm{Na} / \mathrm{K}$ of ions seretion with adding $3 \mathrm{mmol} \mathrm{L} \mathrm{L}^{-1} \mathrm{Na}_{2} \mathrm{SiO}_{3}$ under different $\mathrm{NaCl}$ concentrations

\begin{tabular}{|c|c|c|c|c|c|}
\hline \multirow{2}{*}{$\begin{array}{c}\text { 处理 } \\
\text { Treatment }\end{array}$} & \multirow{2}{*}{$\begin{array}{c}\mathrm{NaCl} \text { 浓度 } \\
\mathrm{NaCl} \text { concentrations (mmol }\end{array}$} & \multicolumn{4}{|c|}{$\mathrm{Na} / \mathrm{K}$} \\
\hline & & $\begin{array}{l}\mathrm{Na} \text { 地上/ } / \mathrm{Na} \text { 地下 } \\
\mathrm{Na}_{\text {shoot }} / \mathrm{Na}_{\text {root }}\end{array}$ & $\begin{array}{l}\text { 叶片 } \\
\text { Leaves }\end{array}$ & $\begin{array}{c}\text { 根 } \\
\text { Roots }\end{array}$ & $\begin{array}{l}\text { 盐分分泌液 } \\
\text { Secreted fluid }\end{array}$ \\
\hline$-\mathrm{Si}$ & 0 & $0.637 \pm 0.031$ & $0.075 \pm 0.009$ & $0.165 \pm 0.011$ & $0.321 \pm 0.046$ \\
\hline$-\mathrm{Si}$ & 60 & $1.421 \pm 0.082$ & $0.439 \pm 0.031$ & $0.350 \pm 0.056$ & $7.12 \pm 0.21$ \\
\hline$-\mathrm{Si}$ & 120 & $0.876 \pm 0.066$ & $0.520 \pm 0.09$ & $0.883 \pm 0.051$ & $9.88 \pm 0.173$ \\
\hline$+\mathrm{Si}$ & 0 & $0.712 \pm 0.05$ & $0.097 \pm 0.014$ & $0.174 \pm 0.009$ & $0.27 \pm 0.017$ \\
\hline$+\mathrm{Si}$ & 60 & $0.905 \pm 0.079$ & $0.310 \pm 0.019$ & $0.468 \pm 0.049$ & $2.882 \pm 0.096$ \\
\hline$+\mathrm{Si}$ & 120 & $1.061 \pm 0.089$ & $0.642 \pm 0.051$ & $0.824 \pm 0.041$ & $6.993 \pm 0.144$ \\
\hline
\end{tabular}

3 次测定结果平均值 \pm 标准误差 Average of 3 replications Means \pm SE $(n=3)$

表 4 硅盐互作对小獐毛叶片和根部可溶性蛋白及 POD 活性的影响

Table 4 The effect of silicon on the content of soluble protein and POD activity of the leaves and roots under salt stress

\begin{tabular}{|c|c|c|c|c|c|}
\hline \multirow{2}{*}{$\begin{array}{c}\text { 处理 } \\
\text { Treatment }\end{array}$} & \multirow{2}{*}{$\begin{array}{c}\mathrm{NaCl} \text { 浓度 } \\
\mathrm{NaCl} \text { concentration } \\
\left(\mathrm{mmol} \mathrm{L} \mathrm{L}^{-1}\right)\end{array}$} & \multicolumn{2}{|c|}{ 可溶蛋白 Soluble protein ( $\left.\mathrm{mg} \mathrm{g}^{-1} \mathrm{Dw}\right)$} & \multicolumn{2}{|c|}{ POD 比活性 The activity of $\operatorname{POD}\left(\mu \mathrm{mg}^{-1} \mathrm{Pr}\right)$} \\
\hline & & 叶片 Shoots & 根 Roots & 叶片 Shoots & 根 Roots \\
\hline$-\mathrm{Si}$ & 0 & $70.97^{\mathrm{a}}$ & $53.6^{\mathrm{a}}$ & $296.1^{\mathrm{b}}$ & $1849.5^{\mathrm{b}}$ \\
\hline$-\mathrm{Si}$ & 120 & $118.7^{\mathrm{a}}$ & $52.41^{\mathrm{a}}$ & $344.08^{\mathrm{ab}}$ & $2226.5^{\mathrm{ab}}$ \\
\hline$+\mathrm{Si}$ & 0 & $76.05^{\mathrm{a}}$ & $65.48^{\mathrm{a}}$ & $234.86^{\mathrm{b}}$ & $2118.66^{\mathrm{ab}}$ \\
\hline$+\mathrm{Si}$ & 120 & $76.49^{\mathrm{a}}$ & $47.99^{\mathrm{a}}$ & $528.06^{\mathrm{a}}$ & $2342.93^{\mathrm{a}}$ \\
\hline
\end{tabular}

3 次测定结果平均值, 不同字母的处理差异显著 $(p<0.05)$ Average of 3 replications, significance of difference at 0.05 significance level 

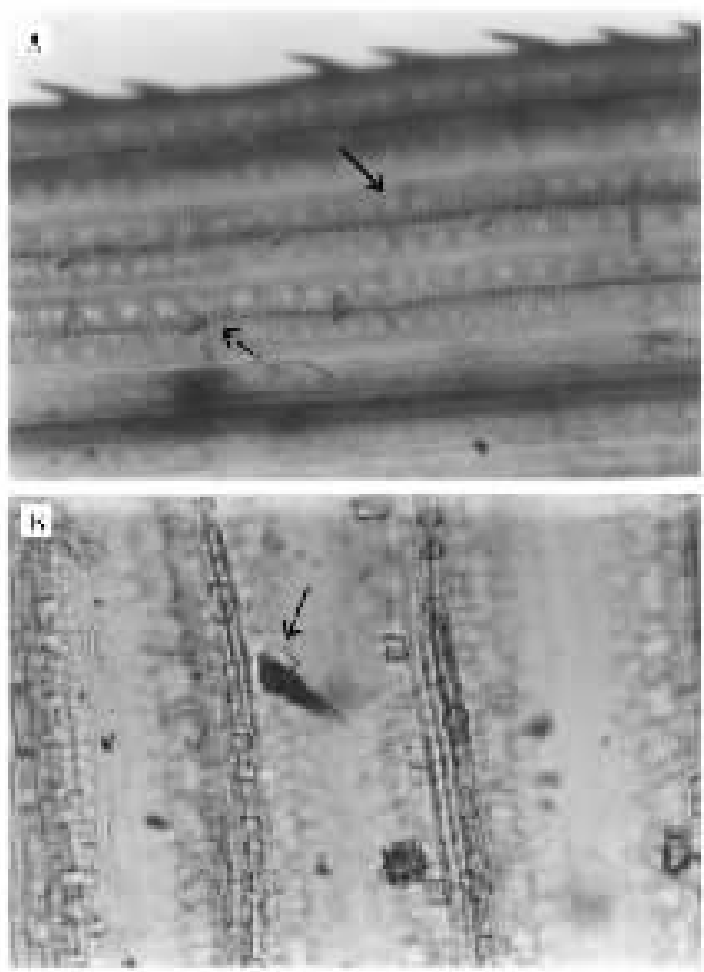

图 3 小獐毛叶表及表皮毛硅元素分布

Fig. 3 Si distribution on leaf surfaces of Aeluropus pungens A 旪表 Leaf surface $(\times 100) \quad$ B 表皮毛 Papillaes $(\times 400)$

$\rightarrow$ 哑铃形硅 Dumbbell-shaped silicon $\quad-\cdots$ 表皮生硅 Popillaes silicon
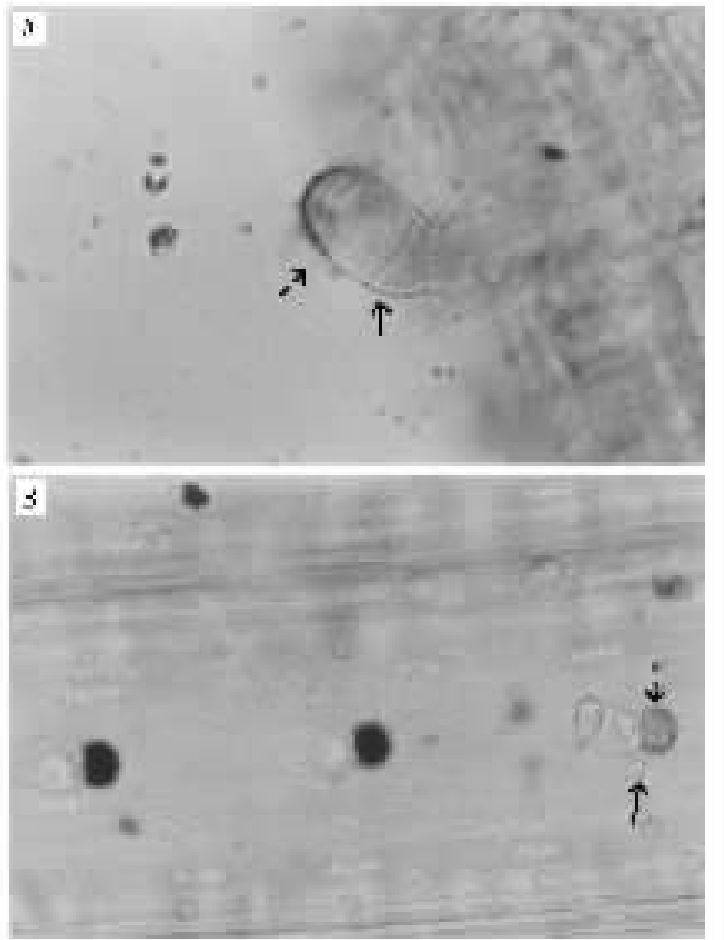

图 4 小獐茅盐腺帽细胞硅元素及 POD 分布

Fig.4 Si distribution and POD activity of the cap cell on salt glands

A 硅元素分布 Si distribution $(\times 1000) \quad$ B :POD 活性 POD activity $(\times 400)$

$\rightarrow$ 基细胞 The base cell $\quad-\quad \rightarrow$ 帽细胞 The cap cell

的渗透调节物质有关。小獐毛生态适应性体现在盐 下可保持稳定的含水量及使叶片维持正常的姿态, 以保证物质及气体交换的顺利进行。2) Marcum (1999) 比较了 6 种草坪草, 发现植物对盐分的耐受 力与其对钠、氯元素排出有关。而离子的排出在很 大程度与离子经盐腺的分泌有关。Pollak 和 Waisel (1979) 认为离子分泌最适条件是低的外界盐分浓度 及较高的大气湿度。本文实验结果显示出盐胁迫有 促进盐腺对于钠离子分泌的作用。同时在盐腺分泌 的离子中 盐腺对钠元素选择性分泌最高。通过对 盐分分泌液及植物体中的 $\mathrm{Na} / \mathrm{K}$ 比较, 显示出盐腺 对钠、钾元素分泌的选择性远高于植物体对于钠、钾 吸收的选择性。因此小獐毛盐腺对钠元素的高分泌 选择性在控制体内盐分方面有一定贡献。3) Rengel (1992)认为钙元素在盐胁迫下有保护膜结构并阻 止钠离子反渗的作用，从而维持膜完整性减少细胞 溶质中钾离子渗出的作用。而小獐毛植物体内钙元 素在盐胁迫下向功能叶分配，对维持矿质平衡，调节 渗透压以及保证正常代谢活动有一定的作用。而硅 元素在叶表的分布可以降低盐胁迫下地上部蒸腾作 用，以保持植物体内水分含量，在降低因供水不足对 植物造成的渗透伤害方面有着积极的意义。4) 与 大多数植物在盐胁迫下表现为抗氧化酶活性的减低 及自由基含量的增加相比较，小獐毛植物体在盐胁 迫下却表现为其抗氧化酶 POD 活性有所增加的现 象。说明其清除自由基的能力增强。因此小獐毛可 通过保持植物体内水分平衡, 减少离子毒害, 加强抗 氧化酶系统功能适应一定程度的盐胁迫。

Wang 和 Maser (1994)推测过氧化氢，过氧化物 酶可能有促进硅沉积的作用, 而纳米硅胶颗粒对过 氧化物酶的活性具有促进作用。通过对硅在盐腺的 分布及 POD 细胞化学定位结果显示, 硅在植物叶表 具有较高程度的分布, POD 活性与硅在帽细胞的沉 积具有很好的一致性。这一方面与小獐毛对硅的富 积及体内较高的含硅量有关, 同时也暗示出硅的沉 积与 POD 活性可能存在一定关系。同时小獐毛对 元素的吸收和分布体现出对硅元素的高累积和对钠 元素低吸收的特点。Yeo 等 (1999)) 认为盐胁迫下 加硅可减少水稻对盐分的吸收及盐分向地上的运 输。实验结果表明,在 $60 \mathrm{mmol} \mathrm{L} \mathrm{L}^{-1}$ 盐浓度下, 使用 硅可有效地减少盐分在叶片中的分布，降低对钠元 素的选择性 增加植物对钾元素的选择性。同时减 少钠元素在地上的分配比例, 但不减少钠元素在根 中的分配。说明硅可通过减少盐分由地下向地上部 
的运输而增加其在低盐浓度下的抗盐性。同时在盐 胁迫下施硅, 可有效地提高小獐毛叶片 POD 活性, 增强叶片消除自由基的能力, 因此对提高植物抗盐 作用有着积极的意义。

近年来, 由于土壤盐渍化、次生盐渍化面积逐年 增加, 加之地球可利用水资源的减少, 对于草坪草灌 溉可考虑应用低品质水资源，同时也需要培养更多 的耐盐品种。而生长于高热、高光强、干旱盐渍化土 壤的小獐毛具有种子易采摘、易萌发、出苗后易培养 成活的特点, 因此这种具有草坪草生长性质的野生 盐生植物的引用 将有助于开发盐碱地生产潜力 缓 解水资源不足, 并具有一定的保护生态环境的意义。 而盐胁迫条件下在培养环境中添加硅营养在促进植 物对硅元素吸收的同时, 可降低盐离子对植物体的 伤害, 这将为盐碱地上引种和驯化有经济价值的盐 生植物以及探索提高作物抗盐途径提供参考。

\section{参 考 文 献}

Ahmad, R., S. H. Zaheer \& S. Ismail. 1992. Role of silicon in salt tolerance of wheat (Triticum aestivum L.). Plant Science, 88:43 $\sim 50$.

Ball, M. C . 1988. Salinity tolerance in the Mangroves aegiceras corniculatum and Avicennia marina I. Water use in relation to growth, carbon partitioning and salt balance. Australian Journal of Plant Physiology, 15:447 464 .

Dayamandan, P. , P. B. Kaufman \& C. I. Franllin. 1983. Dectection of silica in plants. American Journal of Botany, 70:1079 1084 .

Ehman, S.R., P. J.C. Harris \& W.F. Bourne. 1996. The effect of sodium chloride on germination and the potassium and calcium contents of Acacia seeds. Seed Science Technology, 25:45 57 .

Epstin, E. 1999. Silicon. Annual Review of Plant Physiology. Plant Molecular Biology, 50:641 651.

Fitzgerald, M. A., A. D. Orlovich \& G. W. Allaway. 1992. Evidence that abaxial leaf glands are the sites of salt secretion in leaves of the mangrove Avicennia marina (Forsk.) Vireh. New Phytologist, 120:1 $\sim 7$.

Flowers, T.J., P.F.Troke \& A.R. Yeo. 1977. The mechanism of salt tolerance in halophytes. Annual Review of Plant Physiology, 28: $89 \sim 121$.

Flowers, T. J., M. A. Hajibagheri \& N. J. W. Clipson. 1986. Halophytes. Quarterly Review of Biology, 61:313 317 .

Fridlund, S., S. Littlefield \& J. Rivers. 1994. The use of modified microwave digestion/dissolution for the quantitative determination of aluminum, silicon, and iron in biological materials by inductively coupled plasma spectrometry. Communications in Soil Sci- ence and Plant Analysis, 25:933 941.

Hepler, P. K. \& M. R. Rice. 1972. Cytochemical localization of peroxidase activity in wound vessel members of coleus. Canadian Journal of Botany, 50:977 983.

Hernandez, J.A., F. J. Corpass, M. Gomez \& L. A. Rio. 1993. Salt -induced oxidative stress mediated by activity oxygen species in pen leaf mitochondria. Physiolgia Plantarum,89:103 110 .

Liang, Y. C. 1999. Effects of silicon enzyme activity and sodium. potassium and calcium concentration in barley under salt stress. Plant and Soil, 209:217 224.

Marcum, K. B. \& C. L. Murdoch. 1990. Salt glands in zoysiease. Annals of Botany, 66:1 7 .

Marcum, K. B. \& C. L. Murdoch. 1990. Growth responses ion relation, and osmotic adaptations of eleven $\mathrm{C}_{4}$ turfgrasses to salinity. Agronomy Journal, 82:892 896 .

Marcum, K. B. 1999. Salinity tolerance mechanisms of grasses in the subfamily chloridoideae. Crop Science, 39:1153 1160.

Marcum, K. B. 1994. Salinity tolerance mechanisms of six $C_{4}$ turfgrasses. Jounal of American Society \& HortScience, 119: 779 784 .

Mensen, R., A. Hager \& P. Salzer. 1998. Elicitor-induced changes of wall-bound and secreted peroxidase activities in suspension-cultured spruce (Picea abies) cells are attenuated by auxins. Physiologia Plantarum,102:539 546.

Pollak, G . \& Y. Waisel. 1979. Ecophysiolgy of salt excretion in Aeluropus litoralis (Graninae). Physiologia Plantarum, 47:177 184.

Rafig, A., S.H.Zaheer \& S. Ismail. 1992. Role of silicon in salt tolerance of wheat (Triticum aestivum L.) . Plant Science, 88:43 $\sim 50$.

Rengel, Z.1992. The role of calcium in salt toxicity. Plant, Cell and Environment, 15:625 632 .

Schilmper, A.F. 1986. Saline environment and plant growth. India: Agro Botanical Publishers. $64 \sim 66$.

Storey, R., W. W. Thomson. 1994. An X-ray microanalysis study of the salt glands and intracellular calcium crystals of Tamarix. Annals of Botany, 73:307 313 .

Wang, J. \& N. Naser. 1994. Improved performance of carbon paste ampermeric biosensors through the incorporation of fumed silica. Electroanalysis, 6:571 575.

Xu, L. L. (徐朗莱) \& M. B. Ye(叶茂炳). 1989. A method for the continous determination of peroxidase activity. Journal of Nanjing Agricultural University (南京农业大学学报), 12(3): $82 \sim 83$. (in Chinese)

Yeo, A. R., S.A. Flowers, G. Rao, K. Welfare, N. Senanayake $\&$ T. J. Flowers. 1999. Silicon reduces sodium uptake in rice $(O$ ryza sativa L. ) in saline conditions and this is accounted for by a reduction in the transpirational bypass flow. Plant, Cell and Environment, 22:559 565.

Zhao, K.F(赵可夫) \& J.Li (李军). 1999. Effect of salinity on the contents of osmotic of monocotyledenous halophytes and their condition to osmotic adjustment. Acta Botanica Sinica (植物学 报), $\mathbf{4 1}: 1287 \sim 1292$. (in Chinese with Englihs abstract) 